\title{
Le rétablissement des migrations sur la rivière Orme (Basse Normandie)
}

\author{
Re-establishment of migrations \\ on the Orne river (Lower Normandy)
}

\author{
A. Richard
}

Conseil supérieur de la pêche

Délégation régionale $\mathrm{n}^{\circ} 2$, Cesson-Sévigné

Dans le cadre des travaux menés sur les salmonidés migrateurs en Basse-Normandie, un programme visant à rétablir leur libre circulation sur l'Orne a été mise en place en 1981, grâce à un large consensus local.

Regroupant diverses actions complémentaires sur les plans techniques, règlementaires et biologiques, il doit permettre de mettre en valeur le cheptel de truites de mer et de saumons de ce cours d'eau.

As part of works carried out on migrating salmonoidea in Lower Normandy, a programme aiming at re-establishing their free circulation in the Orne was set up in 1981, following an extensive local consensus.

Bringing together various additional technical, statutory and biological activities, this should improve the stock of sea-trout and salmon along this river.

\section{Présentation de l'Orne}

Premier fleuve bas normand avec $175 \mathrm{~km}$ de long et un bassin versant de $2900 \mathrm{~km}^{2}$, l'Orne prend sa source au cœur du département du même nom, puis coule vers le nord pour se jeter en baie de Seine après avoir traversé le département du Calvados.

Rivière frontière entre le massif armoricain et le bassin parisien, l'Orne présente un cours varié et un profil en long original: après un parcours d'abord assez lent a travers les plaines sédimentaires de l'amont, elle s'encaisse dans les gorges granitiques de Saint Aubert puis traverse ensuite les schistes et grès de la Suisse Normande en une vallée sinueuse et profonde, avant de retrouver sur ses derniers 25 kilomètres les calcaires de la plaine de Caen.

A mi-parcours se situe donc une importante zone de rupture de pente, tandis que le cours aval dans le Calvados est ralenti par une trentaine de barrages, en activité (microcentrales) ou abandonnés.

Le module de l'Orne est de $25 \mathrm{M}^{3} / \mathrm{s}$ à l'exutoire. En raison de la nature géologique de son bassin versant 
dominé par les terrains primaires, l'Orne présente un régime contrasté, aux débits élevés en saison humide (jusqu'à $150-200 \mathrm{M}^{3} / \mathrm{s}$ ), mais faibles en période d'étiage $\left(2,5\right.$ à $\left.3 \mathrm{M}^{3} / \mathrm{s}\right)$.

Trois de ses cinq principaux affluents, à savoir la Rouvre, la Baize et le Noireau, la rejoignent en aval des gorges de St Aubert, aux confins des deux départements.

\section{Les populations de salmonidés migrateurs}

Jusqu'à ces dernières décennies, l'évolution historique des migrateurs de l'Orne correspond à un schéma malheureusement trop connu sur les rivières françaises.

L'Orne fut naguère fréquentée par le saumon atlantique, comme en témoigne un vieux plan de Caen au Xvi siècle. Dans son Traité général des Pêches (1769), Duhamel Du Monceau faisait également état de remontées dans ce cours d'eau. Dès cette époque, la multiplicité des ouvrages établis sur le cours aval, barrages de moulins mais aussi pêcheries, le transformaient en une succession quasi-ininterrompue de biefs. Les principales zones de frayères se situaient alors dans les gorges de St Aubert, ainsi que dans certains affluents tels que la Rouvre, le tout à une centaine de kilomètres de l'estuaire. Néanmoins, la remontée des géniteurs ne paraissait pas à cette époque devoir être entravée du fait de la faible hauteur des ouvrages. A la fin du $\mathrm{XIX}^{e}$ siècle, l'industrialisation de la vallée de l'Orne et la création de filatures ont provoqué l'apparition d'obstacles majeurs, du fait du rehaussement de nombreux barrages en vue d'une meilleure utilisation énergétique de la rivière. Les entraves à la remontée des saumons vers les frayères se sont progressivement multipliées, malgré les plaintes répétées de communes riveraines, alertées par la raréfaction de ce poisson. Le 23 février 1924, l'Orne a été soumise par décret, au régime des échelles à poissons sur 130 kilomètres de son cours, sans que cette mesure ne se soit concrétisée sur le terrain. Les dernières captures significatives semblent dater de la fin des années 20 et en 1934, la construction du barrage infranchissable de Grimbosq à une quarantaine de kilomètres de la mer ferma tout accès vers l'amont.

Privé de l'ensemble de ses frayères, le saumon de l'Orne fut ainsi rapidement condamné à disparaître. Plus récemment, et renforçant ainsi l'aménagement hydroélectrique de la rivière, Electricité de France construisit en 1960 le barrage de Rabodanges sur une partie des gorges de St Aubert, ainsi que le lac de compensation de St Philbert en aval de celles-ci.

Cependant, depuis les années 1960, un phénomène original semble s'être développé sur l'Orne, à savoir l'implantation d'un autre salmonidé migrateur, la truite de mer et ceci à la faveur d'un concours de circonstances favorables dont les principales paraissent avoir été :

- d'une part l'intensification des alevinages en truite commune de souches plus ou moins identifiées, voire nordiques ou anadromes, qui semblent avoir généré des remontées de truites de mer;

- d'autre part, une évolution favorable des habitats sur le cours aval, consécutive à l'abandon de plusieurs barrages et usines hydroélectriques, notamment à la suite de dommages subis lors de la dernière guerre. Plusieurs biefs fort anciens ont ainsi en partie ou totalement disparu aux profits de zones peu profondes et courantes à la granulométrie élevée, souvent propices à la reproduction des truites de mer ou à la production de juvéniles;

- enfin, la réalisation en 1973 d'une passe à poissons fonctionnelle au barrage de May sur Orne, premier obstacle à la remontée, permit de garantir les migrations sur le cours en aval de Grimbosq.

En 1979, une convention d'étude a été passée entre le ministère de l'Environnement et la Fédération départementale des associations agréées de pêche et de pisciculture du Calvados, afin de mieux cerner la situation de la truite de mer dans ce département. A l'issue des premiers résultats, une station de piégeage fut installée en 1981 à la sortie de l'échelle de May/Orne à $23 \mathrm{~km}$ de la mer. Les nombreux renseignements ainsi obtenus ont montré que les remontées de truites de mer sur l'Orne se situaient actuellement, selon les années, de 800 à 2000 individus dont les taille et poids moyens, $62 \mathrm{~cm}$ pour $3 \mathrm{Kg}$, reflètent la forte croissance. Par contre, le saumon atlantique n'est plus représenté que par quelques poissons d'été.

1986 verra l'achèvement de l'ensemble du programme de passes à poissons avec l'équipement de cinq autres barrages, qui, loin d'être infranchissables comme les précédents, constituent néanmoins des freins à la migration en périodes d'eaux moyennes et basses (époque des remontées de truite de mer).

\section{Programme de mise en valeur des migrateurs de l'Orne}

Les résultats enregistrés dès la mise en service du piège de May/orne ont servi de véritable révélateur. En effet, les relevés quotidiens et le bilan des remontées ont permis à beaucoup, au-delà du premier étonnement, de prendre conscience non seulement de la richesse méconnue de la rivière, mais surtout des potentialités écologiques et historiques du bassin.

Le développement du cheptel migrateur passant par l'augmentation des zones de frayères et de production de juvéniles accessibles aux géniteurs, le rétablissement des migrations sur tout le cours de l'Orne a donc été rapidement envisagé, non seulement par les collectivités piscicoles directement concernées, mais aussi à la demande des S.I.V.O.M. de la Vallée de l'Orne, désireux d'adjoindre à leurs activités de "Tourisme vert " la pêche sportive des salmonidés migrateurs.

Un dossier technique complet répondant à cet objectif a été préparé par le C.S.P. avec la collaboration du C.E.M.A.G.R.E.F. Présenté fin 1981 aux collectivités piscicoles et locales, le document "Orne, rivière pilote.. » proposait la réalisation de onze ouvrages de franchissement pour un coût global proche de $2000000 \mathrm{~F}$. le financement retenu pour ce programme était le suivant :

$50 \%$ de la part de l'Etat,

$30 \%$ du C.S.P.

$20 \%$ du Département du Calvados.

Le principe d'une telle participation du département put être acquis à la suite d'une visite des installations de 
May/Orne en pleine période de remontées par la Commission du tourisme du Conseil général, lors d'une journée d'information sur le sujet. En outre, une enquête fut réalisée à la demande de celle-ci sur les impacts touristiques de la pêche des migrateurs par le Comité département du tourisme.

Aucune participation financière n'a été demandée à cette époque aux propriétaires des ouvrages. L'article 428-2 du Code rural ne précisait en effet en aucun cas à qui incombait le coût des échelles (ambigüité levée avec le nouvel article 411). De plus, les trois barrages les plus importants avaient déjà été équipés vers 1965 de passes réalisées selon les directives de l'Administration, mais pourtant bien peu fonctionnelles (problèmes de conception et surtout d'implantation sur les sites).

Par contre, la FDAAPP du Calvados n'accepta la maîtrise d'ouvrage de ces travaux qu'en contrepartie de l'arrêt immédiat des éclusées pratiquées couramment par les microcentrales, ces manœuvres déjà fort préjudiciables pour le milieu aquatique étant également de nature à compromettre le bon fonctionnement des passes à poissons. En outre, les administrations chargées des polices de l'eau et de la pêche mirent rapidement en œuvre les dispositions prévues dans le décret 81.375 du 15 avril 1981 (en application de la loi 80.531 du $15 / 7 / 1980$ relative aux économies d'énergie) afin de régulariser la situation des sept microcentrales situées sur l'Orne de sorte que leur exploitation respecte les mesures de préservation de la rivière et de la faune piscicole indispensables à la mise en œuvre du programme "migrateurs".

Un large consensus s'est ainsi rapidement développé autour de la reconquête de l'Orne par les migrateurs à la suite de la mise en évidence de son cheptel de truite de mer par des études biologiques.

Les éléments marquants de ce processus paraissent avoir été tout d'abord les nombreuses visites d'information à la station de contrôle de May/Orne de la part de l'ensemble des administrations départementales et régionales ainsi que d'élus, mais aussi de tout un public de pêcheurs et profanes découvrant la vie de leur rivière.

Puis, l'unanimité s'étant rapidement faite autour de ce projet, celui-ci fut inscrit au premier rang des opérations à mener dans le cadre du Plan quinquennal de restauration des milieux aquatiques 1981-1986. Bénéficiant de tous les appuis techniques, réglementaires, financiers et politiques (au sens d'une volonté locale affirmée de mener à bien l'opération), le programme "Orne" put être mis en œuvre et démarrer sans délais.

L'animation et la coordination des différentes opérations, tant sur le terrain qu'auprès des nombreux partenaires, furent prises en charge par la Délégation régionale du C.S.P. en appui technique auprès des collectivités piscicoles locales.

\section{Le rétablissement des migrations}

\section{a. Travaux}

Dans de telles conditions, la restauration de l'axe des migrations jusque vers les frayères situées aux confins des départements du Calvados et de l'Orne a pu être réalisée entre 1981 et 1985 par l'aménagement des six barrages infranchissables. Répartis en trois tranches, les travaux ont successivement porté sur :

* en 1981 , le barrage de Grimbosq, P.K. 39 ( $\Delta h=3$ m.) : modification de l'ancienne échelle non fonctionnelle par création d'un bassin de repos et d'une seconde volée de 10 mètres à ralentisseurs de fonds inactifs, en prolongement de la partie à ralentisseurs lachadénèdes (qui se terminait par un angle droit et une chute très difficilement négociables pour les poissons).

* en 1983, les barrages :

- du Hom, P.K. $46(\Delta h=2,80 \mathrm{~m})$ : passe à six bassins successifs,

- de St Rémy, P.K. $58(\Delta h=2 \mathrm{~m}$. $)$ : passe à ralentisseurs plans,

- de la Courbe, P.K. $70(\Delta h=3,80 \mathrm{~m}$.) : passe à ralentisseurs plans comprenant deux volées et un bassin de repos intermédiaire.

* en 1985 , les barrages :

- de Thury Harcourt, P.K. $48(\Delta h=2 \mathrm{~m}$.) : passe à ralentisseurs plans,

- du Pont des Vers, P.K. $83(\Delta h=2 \mathrm{~m}$. $)$ : passe à ralentisseurs plans.

Ces aménagements, dont certains ont effacé les causes historiques de la disparition du saumon de l'Orne (St Rémy et Grimbosq) ont rendu le cours de la rivière à nouveau accessible aux migrateurs sur près de $100 \mathrm{~km}$, sans compter les importants parcours sur des affluents tels que la Rouvre et la Baize.

\section{b. Dispositions complémentaires}

La construction de ces échelles s'accompagne de diverses actions visant à garantir à la fois leur bonne efficacité ainsi que l'accroissement de la production en migrateurs du bassin.

La réalisation, le fonctionnement et l'entretien des dispositifs pré-cités ont donc été repris dans les arrêtés de régularisation des microcentrales. Il en est de même pour les aménagements destinés à garantir les dévalaisons de juvéniles et de géniteurs repartant à la mer, nombreux chez la truite de mer. La présence de ces derniers a conduit à établir dès que nécessaire des goulottes de dévalaison à proximité immédiate des grilles d'admission aux turbines.

Outre la libre circulation, les arrêtés garantissent dorénavant sur chaque site le maintien des équilibres biologiques de la rivière, par l'interdiction des éclusées et l'instauration de débits réservés parfois élevés, conduisant à la mise en place de périodes de chomage. Afin d'éviter l'aménagement d'entreprises hydrauliques nouvelles, l'Orne a été classée "rivière réservée " par le décret du 08/06/84 après proposition du Conseil Général du Calvados.

L'amélioration de la qualité de l'habitat salmonicole principalement sur les zones de frayères, a été entreprise sur la Rouvre et l'Orne dans le cadre d'opérations de nettoyages sous l'égide des Fédérations et A.P.P. (chantiers de jeunes, TUC,), également relayées par les travaux de clubs de canoë-kayak. Dans le cadre de la convention EDF-Ministère de l'Environnement, la restauration du potentiel salmonicole des "gorges de St Aubert " enclavées entre les deux barrages EDF et actuellement soumises à des conditions hydrauliques critiques, est à l'étude. La 
mise en service de l'écluse Borlaud du barrage de compensation de St Philbert est prévue pour le printemps 1986. Une campagne de mesures des habitats en fonction de différents débits a été réalisée dans les gorges à l'automne 1985 en vue de la définition d'un débit réservé susceptible de rétablir le potentiel de production de juvéniles de ce secteur de $12 \mathrm{~km}$.

La restauration du stock de saumon atlantique a également été entreprise, symbole de la reconquête de l'Orne. En plus de l'aspect historique, le saumon parait en effet le plus apte à valoriser les importantes frayères situées à une centaine de kilomètres de la mer en y accédant à la faveur des importants débits printanniers de l'Orne. Les FDAPP du Calvados et de l'Orne se sont donc associées dans un effort commun de repeuplement. Après un premier retour d'adultes issus d'une opération de production extensive de smolts limitée à un ruisseau-pépinière, un deversement de 200000 alevins a été effectué début 1984 dans des secteurs d'habitats favorables.

La souche d'importation utilisée devrait permettre d'obenir le retour de saumons de printemps de gros gabarits et combler ainsi l'absence actuelle de remontées de migrateurs pendant tout le début de l'année, les truites de mer n'arrivant qu'en juin. Le repeuplement en jeunes saumons doit encore se poursuivre plusieurs années en faisant notamment appel à des œufs de la souche voisine de la Sélune, captées à la station salmonicole de Cerisel.

\section{c. Perspectives}

La mise en valeur des migrateurs sur l'Orne comprend un nécessaire développement de la ressource s'appuyant principalement sur le rétablissement intégral des migrations, ains que la définition et la mise en œuvre d'une réelle gestion des populations issues de ces programmes.

A cet effet, le suivi des remontées doit être poursuivi à la station de May-sur-Orne de manière à pouvoir mesurer l'évolution des populations de truites de mer et saumons consécutive aux diverses actions de reconquête.
Parallèlement, la recolonisation du bassin fera l'objet d'un suivi attentif sur les zones de frayères et de recrutement à travers le contrôle des migrations et de la reproduction des adultes et l'étude des peuplements de juvéniles. Quant à l'exploitation, l'indispensable connaissance des captures sera fournie par la déclaration des prises prévue dans les nouveaux textes réglementaires sur la pêche fluviale, tandis que la concertation engagée avec le Domaine maritime à ce sujet ne saurait tarder à aboutir.

Outre ces aspects techniques, biologiques et halieutiques, le programme "Orne " est appelé à servir également de support à une large initiation à l'environnement aquatique d'eau douce. La mise en place d'un « Itinéraire de découverte du milieu aquatique " et l'aménagement grand public de la station de May/Orne en 1986 permettront avec l'aide de l'association " Animation Nature dans le Calvados » de faire découvrir à tous la rivière, à la suite des migrateurs.

\section{Conclusion}

Étape essentielle du programme " migrateurs " sur l'Orne, le rétablissement en quelques années des migrations sur ce cours d'eau s'intègre dans un contexte global de reconquête du milieu par les truites de mer et saumons favorisés par un large consensus.

L'articulation des opérations menées par les différents intervenants dans un indispensable souci de chronologie et de complémentarité paraît pouvoir garantir les résultats à la hauteur des motivations et des efforts consentis. A ce titre, l'ensemble des actions engagées sur l'Orne en faveur du renouveau des migrateurs peut constituer un exemple de réalisation pour un cours d'eau de moyenne dimension. A un niveau régional, ce programme a ainsi déjà permis de susciter une dynamique en faveur de la mise en valeur d'autres rivières à migrateurs comme la Vire et la Touques.

\section{Discussion}

Président : M. P. MALAPERT

M. LEYNAUD. - A-t-on essayé de quantifier l'impact de la réussite d'un tel programme des points de vue économique et touristique?

M. RICHARD. - «On n'a pas encore pu le faire...; il faut attendre l'accomplissement d'un cycle biologique, ou même de plusieurs, pour savoir si il y a évolution ". Mais on s'attachera à chiffrer les retombées des actions au niveau de l'état du stock et au niveau des impacts induits qu'on peut en attendre en ce qui concerne la pêche sportive.

Notons que le Calvados est à $200 \mathrm{~km}$ de Paris et que l'un des projets des élus est d'offrir aux amateurs de pêche de bonnes conditions de captures des poissons migrateurs.

M. MASSON. - Dans ma région il y a beaucoup de mortalité sous les barrages, dues aux éclusées essentiellement.
Monsieur RICHARD nous a indiqué que la Fédération du Calvados avait accepté la maîtrise d'ouvrage de certains travaux en contre-partie de l'arrêt immédiat des éclusées pratiquées. Est-il possible de modifier ce type de fonctionnement quelle que soit l'installation? Je voudrais savoir comment un consensus a pu être trouvé avec les exploitants.

M. RICHARD. - Dans le cas particulier de l'Orne, ces éclusées étaient pratiquées l'été, en infraction à la réglementation. Il n'a pas été très difficile de les interdire en obtenant un engagement écrit des usiniers, dans la mesure où cela ne représentait qu'une proportion de gain très faible par rapport aux gains annuels fournis par l'exploitation des microcentrales (entre 1 à $4 \%$ selon les sites concernés). Cette "perte» de gains illégaux a été facilement consentie, par rapport au coût des échelles à poisson.

La réalisation des passes a pu se faire rapidement grâce à l'appui des élus et grâce au consensus dont j'ai parlé. 